\title{
Perda de calor por evaporação cutânea associada às características morfológicas do pelame de cabras leiteiras criadas em ambiente tropical ${ }^{1}$
}

\author{
Elaine Cristina Ligeiro ${ }^{2}$, Alex Sandro Campos Maia ${ }^{3}$, Roberto Gomes da Silva ${ }^{4}$, Cintia Maria \\ Battiston Loureiro ${ }^{5}$
}

\footnotetext{
${ }^{1}$ Financiada pelo Conselho Nacional de Pesquisas e Desenvolvimento Tecnológico (CNPq).

2 Pós-Graduação em Zootecnia, Laboratório de Bioclimatologia, Departamento de Zootecnia, Faculdade de Ciências Agrárias e Veterinárias (FCAV), Universidade Estadual Paulista (UNESP), 14884-900 Jaboticabal, SP.

3 Pós-Graduação em Zootecnia, Laboratório de Bioclimatologia, Departamento de Zootecnia, FCAV/UNESP, 14884-900 Jaboticabal, SP.

${ }^{4}$ Laboratório de Bioclimatologia, Departamento de Zootecnia, FCAV/UNESP, 14884-900 Jaboticabal, SP.

5 Pós-Graduação em Zootecnia, Laboratório de Bioclimatologia, Departamento de Zootecnia, FCAV/UNESP, 14884-900 Jaboticabal, SP.
}

RESUMO - A perda de calor por evaporação cutânea e as características morfológicas do pelame (espessura da capa, comprimento médio dos pêlos, número de pêlos por unidade de área e diâmetro médio dos pêlos) foram avaliadas em 254 cabras das raças Saanen, Alpina e mestiças 1/2 Boer 1/2 Saanen. Utilizou-se uma cápsula ventilada para medir a evaporação cutânea, determinada em três regiões do corpo (próximo ao pescoço, meio do flanco e quarto traseiro), enquanto as características morfológicas do pelame foram avaliadas a partir de amostras de pêlos, tomadas $18 \mathrm{~cm}$ abaixo da coluna vertebral, exceto a espessura da capa, que foi medida in situ. Os resultados indicaram que os animais apresentam pelame pouco denso, formado por pêlos finos e compridos. Animais mestiços ( $1 \frac{1}{2}$ Bôer $1 / 2$ Saanen) apresentaram taxas de evaporação cutânea mais elevadas que os puros das raças Saanen e Alpina.

Palavras-chave: caprinos leiteiros, evaporação cutânea

\section{Heat loss by cutaneous evaporation and its association with hair coat characteristics in dairy goats managed in a tropical environment}

\begin{abstract}
Heat loss by cutaneous evaporation and hair coat characteristics (coat thickness, hair length, number of hairs per unit area, and hair diameter) were measured in 254 Saanen, Alpine, and crossbred ( $1 / 2$ Boer $1 / 2$ Saanen) goats. The hair coat thickness was measured just below the dorsal line of thorax followed by collection of hair samples using adapted pliers. Cutaneous evaporation was measured by a ventilated capsule technique in three regions (neck, thorax center, and hind leg) that were assumed to be representative of the average rate of cutaneous evaporation from the whole skin surface. Goats showed low density hair coats constituted by thin and long hair. Heat loss measured by cutaneous evaporation was lower than that reported in cattle; crossbred animals ( $1 / 2$ Boer $1 / 2$ Saanen) had greater rates of cutaneous evaporation than the purebred Saanen and Alpine goats.
\end{abstract}

Key Words: dairy goats, cutaneous evaporation

\section{Introdução}

Em elevados níveis de temperatura ambiente, a evaporação torna-se a principal via para a dissipação de energia térmica dos animais (Finch, 1985; Gebremedhin et al., 1981), a qual ocorre na superfície da epiderme, pela sudação (McLean, 1963b; Taneja, 1958, 1959; daSilva \& Staling, 2003; Maia et al., no prelo), e no trato respiratório (Stevens, 1981; daSilva et al., 2002; Maia et al., 2005). Por outro lado, sob essas condições, a condução, a convecção e a radiação podem eventualmente tornar-se mecanismos de ganho de energia térmica, pois dependem diretamente da temperatura ambiente (McLean, 1963b; Gebremedhin et al., 2001; daSilva, 2000; Maia et al., no prelo).
A perda de calor por evaporação em bovinos e ovinos ocorre principalmente no nível da epiderme, respondendo por, aproximadamente, $80 \%$ da perda total (McLean 1963a; Finch, 1985; Maia et al., no prelo; da Silva \& Staling, 2003). No entanto, a evaporação sobre uma epiderme coberta por pêlos não ocorre da mesma forma que em uma superfície lisa e exposta, pois, acima da epiderme existe uma camada de pelame à qual adiciona resistência à difusão do vapor. Sabe-se que pelames bem assentados e pouco densos possuem menor resistência à transferência de vapor que aqueles densos e espessos (Cena \& Montheith, 1975ab), os quais possibilitam maior taxa de transferência de energia térmica resultante da sudação - ocorre na superfície da epiderme para sua vizinhança, em razão da menor resistência à difusão 
do vapor (Cena \& Monteith, 1975b) - e, conseqüentemente, adaptam-se melhor às elevadas temperaturas e ao excesso de radiação em ambiente tropical (Scheleger \& Turner, 1965).

A seleção de animais com altas taxas de sudação, aliadas a um pelame com menor resistência à convecção e à difusão de vapor, além de uma epiderme altamente pigmentada (daSilva et al.2003), é um recurso óbvio para o melhoramento de animais para criação em baixas latitudes. Diante do exposto, o objetivo com este trabalho foi obter maiores informações sobre a evaporação cutânea e sua associação às características morfológicas do pelame de cabras leiteiras criadas em ambiente tropical.

\section{Material e Métodos}

Foram utilizadas 254 cabras das raças Saanen e mestiços $1 / 2$ Boer $1 \frac{1}{2}$ Saanen, provenientes de um rebanho da UNESP Jaboticabal, SP, e cabras das raças Alpina e Saanen de outro rebanho localizado em São José do Rio Preto, SP. Os dados referentes aos animais foram obtidos do programa de controle produtivo e reprodutivo em caprinos (PROCAPRI) e as medições foram realizadas durante os meses de janeiro e fevereiro de 2004.

As características morfológicas do pelame estudadas foram: espessura da capa $(\mathrm{E}, \mathrm{mm})$, comprimento médio dos pêlos $(\mathrm{C}, \mathrm{mm})$, número de pêlos por unidade de área $(\mathrm{N}$, pêlos $\left./ \mathrm{cm}^{2}\right)$ e diâmetro médio dos pêlos $(\mathrm{D}, \mu \mathrm{m})$.

A espessura da capa foi medida in situ, $18 \mathrm{~cm}$ abaixo da coluna cervical, utilizando-se uma régua metálica graduada em milímetros provida de um cursor, conforme daSilva (2000). A régua era introduzida perpendicularmente na superfície do pelame até tocar suavemente a pele e o cursor era movido até tocar a superfície externa do pelame. A amostragem de pêlos era realizada na mesma região corporal, utilizando-se um alicate adaptado, conforme Lee (1953). A partir destas amostras, foram avaliadas as demais características $(\mathrm{C}, \mathrm{N}$ e D).

Para determinação do comprimento médio dos pêlos, foi considerada a média aritmética do comprimento dos dez maiores pêlos, selecionados por uma análise visual da amostra e medidos com paquímetro, segundo o procedimento recomendado por Udo (1978). O número de pêlos por unidade de área foi determinado por contagem direta do número de pêlos da amostra, correspondente à área de $18 \mathrm{~mm}^{2} \mathrm{da}$ abertura entre as mandíbulas do alicate. Para o diâmetro médio dos pêlos, foi considerada a média aritmética dos diâmetros dos dez maiores pêlos de cada amostra, medidos com um micrômetro digital Mitutoyo, conforme Maia et al. (2003).
As variáveis ambientais temperatura radiante média $\left(\mathrm{T}_{\mathrm{RM}}, \mathrm{K}\right)$, temperatura do $\operatorname{ar}\left(\mathrm{t}_{\mathrm{A}} ;{ }^{\circ} \mathrm{C}\right)$, umidade relativa do ar $\left(\mathrm{U}_{\mathrm{R}}, \%\right)$ e velocidade do vento $\left(\mathrm{U} ; \mathrm{m} \cdot \mathrm{s}^{-1}\right)$ foram registradas continuamente ao longo do dia, de modo que cada observação era referente ao animal amostrado. A temperatura e umidade do ar foram determinadas por meio da leitura da temperatura dos bulbos seco e úmido de um psicrômetro portátil, composto por dois termômetros iguais, um deles com o bulbo envolto por uma luva de tecido de algodão, mantida úmida por meio de uma mecha mergulhada em um frasco de água destilada. Este termômetro fornece temperatura do bulbo úmido e o outro, a temperatura do bulbo seco (daSilva, 2000). A velocidade do ar foi medida com um termoanemômetro (Alnor APM-360) e a temperatura radiante média foi estimada conforme daSilva (2000), utilizando-se a temperatura do globo negro colocado próximo aos animais.

Na Figura 1 está representado o esquema da cápsula ventilada utilizada para medir a evaporação cutânea, a qual foi desenvolvida por Maia et al. (no prelo), com base no trabalho de McLean (1963a), conforme o seguinte esquema de funcionamento: no orifício de saída de ar da cápsula(D), é conectada uma mangueira plástica, pela qual o ar do interior da cápsula é aspirado com o auxílio de um compressor (G) e levado até o analisador de $\mathrm{CO}_{2} / \mathrm{H}_{2} \mathrm{O}(\mathrm{F})$; se o registro $\mathrm{I}_{1}$ estiver aberto e $\mathrm{o}$ registro $\mathrm{I}_{2}$ estiver fechado, é dada a pressão parcial de vapor do ar que sai da cápsula ventilada $\left[\mathrm{P}_{\mathrm{p}}\left\{\mathrm{t}_{\mathrm{CV}}\right\}, \mathrm{kPa}\right]$; caso contrário, é dada a pressãoparcial de vapor da atmosfera $\left[\mathrm{P}_{\mathrm{p}}\left\{\mathrm{t}_{\mathrm{A}}\right\}, \mathrm{kPa}\right]$.

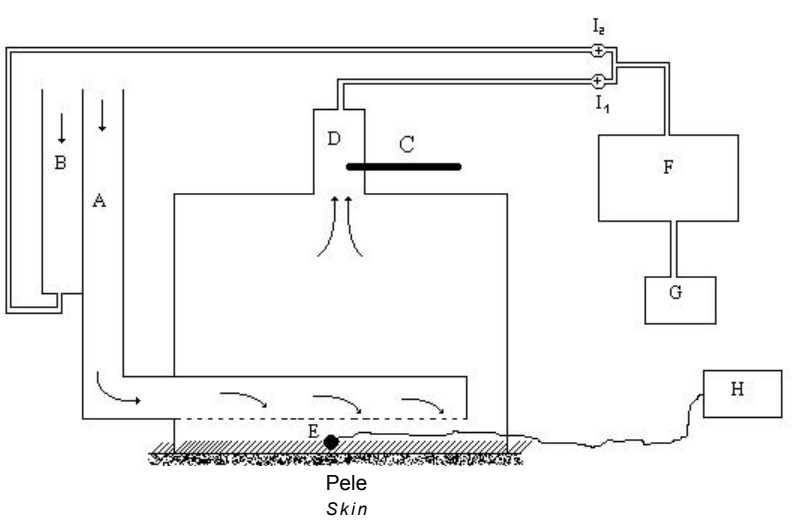

Figura 1 - Cápsula ventilada utilizada neste estudo. Constituída de plástico, com $7,0 \mathrm{~cm}$ de diâmetro e $6,3 \mathrm{~cm}$ de profundidade. A - entrada de ar, B - tubo de amostragem de ar da atmosfera, C - ponta de prova de um termo-anemômetro, D - tubo de saída de ar, $\mathrm{E}$ - ponta de prova de um termômetro, $\mathrm{F}$ - analisador de $\mathrm{CO}_{2} / \mathrm{H}_{2} \mathrm{O}, \mathrm{G}$ - compressor de ar, $\mathrm{H}$ - termômetro de infravermelho e $\mathrm{I}$ = registros.

Figure 1 - Ventilated capsule used in this study made of plastic and containing $7.0 \mathrm{~cm}$ diameter and $6.30 \mathrm{~cm}$ depth. A - air inlet, $B$-tube for sampling atmospheric air, $C$ - probe of a precision thermo-anemometer, $D$ - air outlet tube, E-RTD temperature probe, $\mathrm{F}-\mathrm{CO}_{2} / \mathrm{H}_{2} \mathrm{O}$ gas analyzer, $\mathrm{G}$-air pump, $\mathrm{H}$ - non-contact infrared thermometer and $I$ - registers. 
A quantidade de água evaporada ( $\varepsilon$, g.s. $\left.\mathrm{s}^{-1} \cdot 0,003848 \mathrm{~m}^{-2}\right)$ relativa à área compreendida pela cápsula foi calculada por:

$$
\varepsilon=\mathrm{f}_{\mathrm{C}}\left(\Psi_{\mathrm{CV}}-\Psi_{\mathrm{A}}\right)
$$

em que $\psi_{\mathrm{CV}}$ e $\psi_{\mathrm{A}}$, $\left(\right.$ g.m $\left.{ }^{-3}\right)$ são a umidade absoluta do ar saindo da cápsula ventilada e a do ar da atmosfera,

$$
\begin{aligned}
& \Psi_{\mathrm{A}}=\frac{2166,87 \mathrm{P}_{\mathrm{p}}\left\{\mathrm{t}_{\mathrm{A}}\right\}}{\mathrm{T}_{\mathrm{A}}} \\
& \Psi_{C l}=\frac{2166,87 \mathrm{P}_{\mathrm{p}}\left\{\mathrm{t}_{\mathrm{CV}}\right\}}{\mathrm{T}_{\mathrm{CV}}}
\end{aligned}
$$

e $\mathrm{T}_{\mathrm{A}} \mathrm{eT}_{\mathrm{AC}}$ são a temperatura da atmosfera e do ar que passa através da cápsula, em graus Kelvin, respectivamente.

O fluxo de ar através da cápsula ventilada $\left(\mathrm{f}_{\mathrm{c}}, \mathrm{m}^{3} \cdot \mathrm{s}^{-1}\right)$ foi dado por:

$$
\mathrm{f}_{\mathrm{c}}=\left(\pi r^{2}\right) \mathrm{U}_{\mathrm{cV}}
$$

em que ré o raio geométrico do tubo de entrada do ar da cápsula ventilada $(0,01075 \mathrm{~m})$. Assim $\mathrm{f}_{\mathrm{c}}=3,6305 \times 10^{-4} \mathrm{U}_{\mathrm{cV}}$, sendo $\mathrm{U}_{\mathrm{CV}}\left(\mathrm{m} . \mathrm{s}^{-1}\right)$ a velocidade do ar que passa através da cápsula. $\mathrm{U}_{\mathrm{CV}} \mathrm{eT}_{\mathrm{CV}}$ foram medidas com a ponta-de-prova de um termoanemômetro de precisão (Alnor APM-360) inserido no tubo de saída da cápsula $\mathrm{C}$. $\mathrm{U}_{\mathrm{AC}}$ era regulado para que o $\mathrm{f}_{\mathrm{C}}$ fosse suficiente para manter o gradiente de temperatura $\left(\Delta t=t_{S C}-t_{S}\right)$ próximo de zero, pois, dependendo do valor do $\mathrm{f}_{\mathrm{C}}$ a temperatura da superfície do pelame no interior da cápsula $\left(\mathrm{t}_{\mathrm{SC}},{ }^{\circ} \mathrm{C}\right)$ podia ser alterada, afastando-se daquela da superfície do pelame exterior à cápsula $\left(\mathrm{t}_{\mathrm{S}},{ }^{\circ} \mathrm{C}\right)$ e modificando a troca de calor sensível e a necessidade do resfriamento evaporativo na área de teste (McLean, 1963a), o que não validaria as estimativas.

A temperatura da superfície do pelame no interior da cápsula $\left(\mathrm{t}_{\mathrm{SC}},{ }^{\circ} \mathrm{C}\right)$ foi medida por meio da ponta-de-prova de um termômetro de infravermelho (Rynger ${ }^{\circledR} \mathrm{ST}^{\mathrm{TM}}$ ) inserido no interior da cápsula. A temperatura da superfície do pelame externa à cápsula $\left(\mathrm{t}_{\mathrm{S}},{ }^{\circ} \mathrm{C}\right)$ era medida diretamente pelo termômetro de infravermelho, próximo à mesma área escolhida para medida da evaporação cutânea.

A perda de calor por evaporação foi medida em três regiões diferentes da superfície cutânea: no tronco, aproximadamente $18 \mathrm{~cm}$ abaixo da coluna vertebral; na tábua do pescoço e no quarto traseiro. Essas regiões foram escolhidas com base nos resultados experimentais obtidos por McLean (1963ab), uma vez que a secreção de suor e a evaporação não são homogeneamente distribuídas em toda superfície cutânea. Assim, o fluxo de calor latente na superfície corporal $\left(\mathrm{Ec}, \mathrm{W} \cdot \mathrm{m}^{-2}\right)$ foi determinado por:

$$
\mathrm{E}_{\mathrm{c}}=\frac{\lambda \bar{\varepsilon}}{0,003848}
$$

em que $\lambda$ é o calor latente de vaporização da água $\left(\lambda=2500,7879-2,37374 \mathrm{t}_{\mathrm{A}}, \mathrm{J}_{\mathrm{g}} \mathrm{g}^{-1}\right)$ e $\bar{\varepsilon}\left(\mathrm{g} \cdot \mathrm{h}^{-1} \cdot 0,003848 \mathrm{~m}^{-2}\right)$, a quantidade média de água evaporada nas três diferentes regiões avaliadas.

Os dados foram analisados pelo método dos quadrados mínimos (Harvey, 1960), utilizando-se o procedimento GLM do SAS (SAS, 1998), conforme Littell et al. (1991). Os modelos estatísticos adotados foram:

Modelo 1:

$\mathrm{Y}_{\mathrm{ijk}}=\alpha+\mathrm{L}_{\mathrm{i}}+\mathrm{R}_{\mathrm{ij}}+\mathrm{b}_{1} \mathrm{I}+\mathrm{b}_{2} \mathrm{t}_{\mathrm{A}}+\mathrm{b}_{3} \mathrm{~T}_{\mathrm{RM}}+\mathrm{b}_{4} \mathrm{U}_{\mathrm{R}}+\varepsilon_{\mathrm{ijk}}$ em que $Y_{\mathrm{ijk}}$ é a observação da perda de calor por evaporação cutânea da $k$-ésima cabra; $\mathrm{L}_{\mathrm{i}}$, o efeito fixo do $i$-ésimo local de coleta $(\mathrm{i}=1,2) ; \mathrm{R}_{\mathrm{ij}}$, o efeito fixo daj-ésima raça dentro do $i$-ésimo local de coleta; $b_{1}, b_{2}, b_{3}$ e $b_{4}$, os coeficientes de regressão linear sobre a idade do animal, temperatura do ar, temperatura radiante média e umidade relativa do ar, respectivamente; $\varepsilon_{\mathrm{ijk}}$, o resíduo, incluindo o erro aleatório; e $\alpha$, o intercepto.

Modelo 2:

$\mathrm{Y}_{\mathrm{ijk}}=\alpha+\mathrm{L}_{\mathrm{i}}+\mathrm{R}_{\mathrm{ij}}+\mathrm{b}_{1} \mathrm{I}+\mathrm{b}_{2} \mathrm{t}_{\mathrm{A}}+\mathrm{b}_{3} \mathrm{~T}_{\mathrm{RM}}+\mathrm{b}_{4} \mathrm{U}_{\mathrm{R}}+\mathrm{b}_{5} \mathrm{C}+\varepsilon_{\mathrm{ijk}}$ em que $\mathrm{Y}_{\mathrm{ijk}}$ é a espessura da capa observada na $k$-ésima cabra e $b_{5}$, o coeficiente de regressão linear sobre o comprimento médio dos pêlos. Os demais efeitos incluídos no modelo foram definidos no modelo 1.

Modelo 3:

$$
\mathrm{Y}_{\mathrm{ijk}}=\alpha+\mathrm{L}_{\mathrm{i}}+\mathrm{R}_{\mathrm{ij}}+\mathrm{b}_{1} \mathrm{I}+\varepsilon_{\mathrm{ijk}}
$$

em que $Y_{\mathrm{ijk}}$ é o comprimento médio dos pêlos, o número de pêlos por unidade de área e o diâmetro médio dos pêlos. $\mathrm{L}_{\mathrm{i}}$, $\mathrm{R}_{\mathrm{ij}} \mathrm{e} \mathrm{b}_{1} \mathrm{I}$ foram definidos no modelo 1 .

\section{Resultados e Discussão}

A média geral obtida neste trabalho para a perda de calor por evaporação cutânea (Tabela 1) foi de $72,17 \mathrm{~W} \cdot \mathrm{m}^{-2}$, inferior à de 199,33 W.m-2 ${ }^{-2}$ encontrada por Finch et al. (1982) em bovinos da raça Brahman, com temperatura do ar variando de 27,2 a $35,6^{\circ} \mathrm{C}$; a taxa de perda de calor por evaporação cutânea das cabras foi superior aos $46,14 \mathrm{~W} \cdot \mathrm{m}^{-2}$ determinados por daSilva \& Staling (2003), em ovinos sob temperatura ambiente entre 21,1 e $41,9^{\circ} \mathrm{C}$. Oliveira (2004) estimou taxa de sudação média de $178,8 \mathrm{~g} \cdot \mathrm{h}^{-1} \cdot \mathrm{m}^{-2}$ para caprinos sob temperaturas entre 22,0 e $33,0^{\circ} \mathrm{C}$, o que corresponde a uma perda de calor por evaporação cutânea de, aproximadamente, $119,2 \mathrm{~W} \cdot \mathrm{m}^{-2}$.

Esses valores são de difícil comparação, em razão dos diferentes grupos genéticos avaliados, das diferentes condições ambientais e diferentes metodologias empregadas. Por exemplo, o maior valor registrado por Oliveira (2004) pode ser decorrente da metodologia utilizada, o método colorimétrico de 
Schleger \& Turner (1965), no qual são retirados todos os pêlos dos animais no local da medição, observando-se que a evaporação que se processa no interior da massa de pêlos é menos intensa que a evaporação da superfície cutânea exposta, em termos de calor, eliminado (Kerslake, 1972). Além disso, a taxa de sudação encontrada por Schleger \& Turner (1965) somente foi avaliada próximo ao pescoço, embora a secreção de suor não seja homogênea em toda a superfície corporal (McLean, 1963ab).

As médias da espessura $(5,80 \mathrm{~mm})$ e do comprimento médio dos pêlos $(28,47 \mathrm{~mm}$ ) (Tabela 1$)$ foram superiores às registradas por Maia et al. (2003), em vacas Holandesas $(2,35 \mathrm{~mm}$ de espessura e $12,05 \mathrm{~mm}$ de comprimento para o pelame preto), e por daSilva et al. (1988), em vacas Jersey $(4,11 \mathrm{~mm}$ de espessura e 15,41 $\mathrm{mm}$ de comprimento). Entretanto, as médias do diâmetro $(8,0 \mu \mathrm{m})$ e do número de pêlos por unidade de área (527 pêlos. $\left.\mathrm{cm}^{-2}\right)$ foram inferiores às obtidas por Maia et al. (2003) para os pelames preto $\left(63,62 \mu \mathrm{m}\right.$ e 932 pêlos. $\mathrm{cm}^{-2}$, respectivamente) e branco $\left(60,53 \mu \mathrm{m}\right.$ e 1309 pêlos.cm ${ }^{-2}$, respectivamente), em vacas Holandesas.

Obviamente, estes resultados indicam que os caprinos possuem pelame menos denso que o de bovinos, formado por pêlos finos e compridos, característica amplamente favorável em ambientes quentes, pois permite maior movimentação do ar entre os pêlos, removendo o ar aprisionado no interior da capa. Conseqüentemente, a perda de calor é significativamente afetada na ocorrência de movimentação do ar (convecção forçada) dentro da camada de pêlos, situação que se torna o principal mecanismo de transfe- rência de calor (Gebremedhin et al., 1983; Cena \& Monteith, 1975a), além de diminuir a resistência à transferência de calor por convecção livre, a qual é induzida por um gradiente térmico causado por uma diferença de densidade do ar no interior da capa e, por último, favorece a difusão do vapor através da capa, evaporada na superfície da epiderme para a vizinhança (Cena \& Monteith, 1975b).

Entretanto, quanto maior o diâmetro, maior a condução molecular através dos pêlos e, portanto, maior a condutividade térmica. Assim, pelames constituídos por pêlos grossos seriam mais vantajosos em ambientes quentes que aqueles formados por pêlos finos. No entanto, o acréscimo na transmissão térmica pelo pelame que pode ser atribuído à condução ao longo dos pêlos não é tão significativo (Cena \& Monteith, 1975b), de modo que a convecção livre e a troca radiativa entre os pêlos são os maiores responsáveis pela transferência de calor através do pelame na ausência de movimentação de ar. Caso contrário, a convecção forçada pode dominar este processo, dependendo do nível de movimentação e da posição que o vento atinge a superfície corporal do animal.

$\mathrm{O}$ efeito de local e de raça dentro de local foram significativos $(\mathrm{P}<0,05)$ para perda de calor por evaporação cutânea (Tabela 2), possivelmente em razão da presença dos animais mestiços ( $1 / 2$ Boer $1 / 2$ Saanen) somente no local 1 , visto que apresentaram maior média para essa variável, que diferiu significativamente $(\mathrm{P}<0,05)$ das médias das raças Saanen e Alpina (Tabela 3). Uma hipótese que pode ser considerada para este fato é que o pai dos animais mestiços é da raça Bôer, originária do continente africano. Em geral,

Tabela 1 - Número de observações $(n)$, média geral $(\bar{X})$, erro-padrão $\left(E_{P}\right)$ e valores mínimo e máximo das características morfológicas do pelame, perda de calor por evaporação cutânea e das variáveis ambientais

Table 1 - Number ofobservations $(n)$, mean $(\bar{X})$, standard error $\left(E_{p}\right)$ and minimum and maximum values of the hair coat characteristics, heat loss by cutaneous evaporation, and environmental variables

\begin{tabular}{|c|c|c|c|c|}
\hline $\begin{array}{l}\text { Característica } \\
\text { Item }\end{array}$ & $\mathrm{n}$ & $\bar{X} \pm E p$ & $\begin{array}{l}\text { Mínimo } \\
\text { Minimum }\end{array}$ & $\begin{array}{l}\text { Máximo } \\
\text { Maximum }\end{array}$ \\
\hline $\begin{array}{l}\text { Perda de calor por evaporação cutânea }\left(\mathrm{W} \cdot \mathrm{m}^{-2}\right) \\
\text { Heat loss by cutaneous evaporation }\end{array}$ & 254 & $72,17 \pm 2,32$ & 2,46 & 184,26 \\
\hline $\begin{array}{l}\text { Espessura do pelame }(\mathrm{mm}) \\
\text { Hair coat thickness }\end{array}$ & 254 & $5,80 \pm 0,14$ & 2,50 & 15,00 \\
\hline $\begin{array}{l}\text { Comprimento médio dos pêlos }(\mathrm{mm}) \\
\text { Average hair length }\end{array}$ & 254 & $28,47 \pm 0,33$ & 14,53 & 52,02 \\
\hline $\begin{array}{l}\text { Diâmetro médio dos pêlos }(\mu \mathrm{m}) \\
\text { Average hair diameter }\end{array}$ & 254 & $8,00 \pm 0,11$ & 3,85 & 14,57 \\
\hline $\begin{array}{l}\left.\text { Número de pêlos (pêlos } / \mathrm{cm}^{2}\right) \\
\left.\text { Number of hairs per unit area (hairs } / \mathrm{cm}^{2}\right)\end{array}$ & 254 & $527 \pm 11$ & 139 & 1128 \\
\hline $\begin{array}{l}\text { Temperatura do ar }\left({ }^{\circ} \mathrm{C}\right) \\
\text { Air temperature }\end{array}$ & 254 & 28,30 & 20,0 & 32,60 \\
\hline $\begin{array}{l}\text { Temperatura radiante média }\left({ }^{\circ} \mathrm{K}\right) \\
\text { Mean radiant temperature }\end{array}$ & 254 & 304,22 & 292,66 & 313,74 \\
\hline $\begin{array}{l}\text { Velocidade do vento }\left(\mathrm{m} . \mathrm{s}^{-1}\right) \\
\text { Wind speed }\end{array}$ & 254 & 0,43 & 0,20 & 2,90 \\
\hline $\begin{array}{l}\text { Umidade relativa do ar }(\%) \\
\text { Air humidity }\end{array}$ & 254 & 69,63 & 51,55 & 91,94 \\
\hline
\end{tabular}


animais desenvolvidos nesse continente apresentam maior capacidade de adaptação às condições tropicais, por possuírem melhores mecanismos autônomos de controle térmico, especialmente a sudação.

O coeficiente de regressão da umidade relativa do ar sobre a perda de calor por evaporação cutânea foi significativo $(\mathrm{P}<0,01)$ (Tabela 2$)$ e a relação entre elas foi exponencial e negativa, sendo representada pela equação constante na Figura 2. Entretanto, a correlação entre a umidade e a temperatura do ar foi altamente significativa $(\mathrm{P}<0,0001)$ e negativa $(\mathrm{r}=-0,93)$, de modo que a queda da umidade do ar foi associada à elevação da temperatura do ar e, nessas condições, a capacidade do ar em aceitar vapor aumenta. Conseqüentemente, há maior probabilidade de moléculas de água da superfície do animal escaparem em forma de vapor na direção da atmosfera.

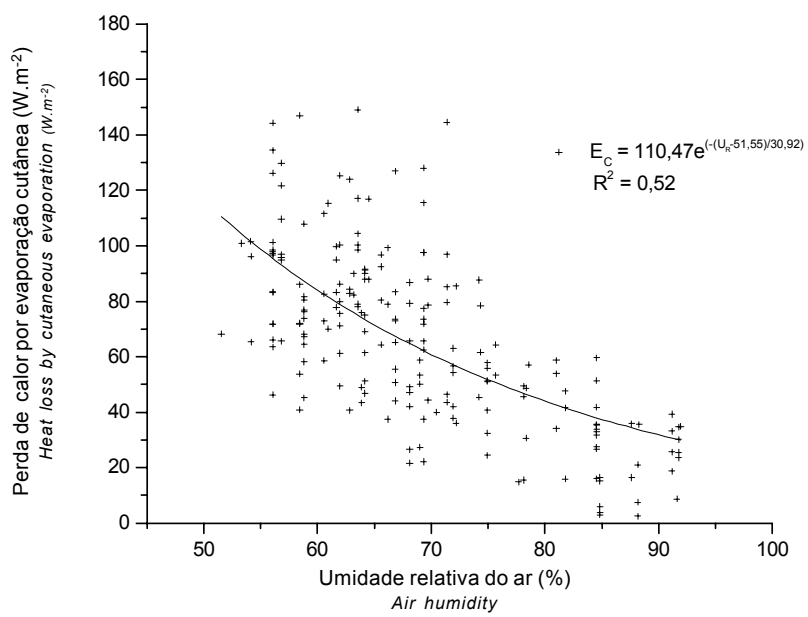

Figura 2 - Efeitos da umidade relativa do ar sobre a perda de calor por evaporação cutânea em cabras das raças Saanen e Alpina.

Figure 2 - Heat loss by cutaneous evaporation in Saanen and Alpine goats as function of the air humidity.

Tabela 2 - Quadrado médio das características morfológicas do pelame e da perda de calor por evaporação cutânea em cabras leiteiras Table 2 - Mean square of the morphological characteristics of coat and heat loss by cutaneous evaporation in dairy goat

\begin{tabular}{|c|c|c|c|c|c|c|}
\hline \multirow[t]{2}{*}{$\begin{array}{l}\text { Fonte de variação } \\
\text { Source of variation }\end{array}$} & \multirow[t]{2}{*}{$\begin{array}{l}\text { g. } 1 . \\
\text { d.f. }\end{array}$} & \multicolumn{5}{|c|}{$\begin{array}{c}\text { Quadrado médio } \\
\text { Mean square }\end{array}$} \\
\hline & & $\mathrm{E}_{\mathrm{C}}$ & $\mathrm{E}$ & $\mathrm{D}$ & $\mathrm{C}$ & $\mathrm{N}$ \\
\hline Local & 1 & $9470,856^{* *}$ & $11,690^{\mathrm{ns}}$ & $440,418^{* *}$ & $75,999^{\mathrm{ns}}$ & $2552,89^{\mathrm{ns}}$ \\
\hline Location & & & & & & \\
\hline $\begin{array}{l}\text { Raça dentro de local } \\
\text { Breed within location } \\
\text { Regressão }\end{array}$ & 2 & $2975,887^{*}$ & $20,166^{*}$ & $2,668^{\mathrm{ns}}$ & $103,552^{*}$ & $111838,30^{*}$ \\
\hline Regression & & & & & & \\
\hline Idade & 1 & $214,543^{\mathrm{ns}}$ & $72,994^{* *}$ & $19,521^{* *}$ & $421,256^{* *}$ & $1586965,4^{* *}$ \\
\hline Age & & & & & & \\
\hline $\mathrm{t}_{\mathrm{A}}$ & 1 & $1601,300^{\mathrm{ns}}$ & $15,898^{\mathrm{ns}}$ & - & - & - \\
\hline $\mathrm{T}_{\mathrm{RM}}$ & 1 & $2975,747^{\mathrm{ns}}$ & $1,872^{\mathrm{ns}}$ & - & - & - \\
\hline $\mathrm{U}_{\mathrm{R}}$ & 1 & $9020,815^{* *}$ & $20,538^{* *}$ & - & - & - \\
\hline $\mathrm{C}$ & 1 & - & $238,743^{* *}$ & - & - & - \\
\hline Resíduo & 244 & 941,572 & 4,340 & 1,399 & 27,49 & 27078,67 \\
\hline
\end{tabular}

$\mathrm{E}_{\mathrm{C}}$ - Perda de calor por evaporação cutânea (heat loss by cutaneous evaporation), E - Espessura da capa (coat thickness), D - Diâmetro médio dos pêlos (average hair diameter), C - Comprimento médio dos pêlos (average hair length), $\mathrm{N}$ - Número de pêlos por unidade de área (number of hair per unit of area), $\mathrm{t}_{\mathrm{A}}$ - temperatura do ar (airtemperature), $\mathrm{T}_{\mathrm{RM}}$ - temperatura radiante média (mean radiant temperature), $\mathrm{U}_{\mathrm{R}}$ - Umidade relativa do ar (air humidity). ${ }^{* *}$ significativo ( $\left.\mathrm{P}<0,01\right)$ significant $(P<0.01)$; "significativo $(P<0,05)$ significant $(P<0.05)$; ${ }^{\text {ns }}$ não-significativo $(P>0,05)$ not significant $(P>0.05)$.

Tabela 3 - Médias estimadas por quadrados mínimos e erro-padrão das características do pelame e da perda de calor por evaporação cutânea em cabras leiteiras, conforme o local e a raça (local)

Table 3 - Least squares means and standard errors of hair coat traits and heat loss by cutaneous evaporation in dairy goats, according to location and breed

\begin{tabular}{|c|c|c|c|c|c|c|}
\hline $\begin{array}{l}\text { Efeito } \\
\text { Effect }\end{array}$ & $\mathrm{n}$ & $\mathrm{E}_{\mathrm{C}}$ & $\mathrm{E}$ & $\mathrm{D}$ & $\mathrm{C}$ & $\mathrm{N}$ \\
\hline \multicolumn{7}{|l|}{ Local } \\
\hline \multicolumn{7}{|l|}{ Location } \\
\hline Jaboticabal & 57 & $86,63^{a} \pm 5,04$ & $5,26^{\mathrm{a}} \pm 0,29$ & $10,49^{a} \pm 0,16$ & $29,47^{\mathrm{a}} \pm 0,71$ & $532^{\mathrm{a}} \pm 22$ \\
\hline S.J.R.Preto & 197 & $67,72^{b} \pm 2,33$ & $5,93^{a} \pm 0,15$ & $7,29^{b} \pm 0,08$ & $28,13^{\mathrm{a}} \pm 0,37$ & $524^{\mathrm{a}} \pm 12$ \\
\hline \multicolumn{7}{|l|}{ Raça dentro local } \\
\hline \multicolumn{7}{|l|}{ Breed within location } \\
\hline Saanen & 25 & $76,66^{\mathrm{ab}} \pm 6,95$ & $4,85^{\mathrm{b}} \pm 0,43$ & $10,75^{\mathrm{a}} \pm 0,24$ & $29,31^{\mathrm{a}} \pm 1,07$ & $543^{a} \pm 34$ \\
\hline $1 / 2$ Boer $1 / 2$ Saanen & 32 & $96,60^{a} \pm 6,23$ & $5,67^{\mathrm{ab}} \pm 0,38$ & $10,24^{\mathrm{a}} \pm 0,21$ & $29,62^{\mathrm{a}} \pm 0,94$ & $522^{\mathrm{a}} \pm 29$ \\
\hline Saanen & 102 & $69,68^{\mathrm{b}} \pm 3,24$ & $6,31^{\mathrm{a}} \pm 0,21$ & $7,39^{b} \pm 0,12$ & $29,16^{\mathrm{a}} \pm 0,52$ & $558^{\mathrm{a}} \pm 16$ \\
\hline Alpina & 95 & $65,75^{b} \pm 3,22$ & $5,56^{\mathrm{ab}} \pm 0,22$ & $7,19^{b} \pm 0,12$ & $27,11^{\mathrm{b}} \pm 0,54$ & $491^{\mathrm{b}} \pm 17$ \\
\hline
\end{tabular}

$\mathrm{n}$ - número de observações (number of observations), $\mathrm{E}_{\mathrm{C}}$ - perda de calor por evaporação cutânea (heat loss by cutaneous evaporation), $\mathrm{E}$ - espessura da capa (coat thickness), D - diâmetro médio dos pêlos (average hair diameter), C - comprimento médio dos pêlos (average hair length), $\mathrm{N}$ - número de pêlos por unidade de área (number of hair per unit of area).

Médias seguidas da mesma letra, para cada efeito e dentro de cada coluna, não difere $(P>0,05)$ pelo teste Tukey.

Means with the same superscript, for each effect and within each column, do not differ $(P>0.05)$ by Tukey test. 


\section{Conclusões}

Os caprinos apresentam pelame pouco denso, formado por pêlos finos e compridos. A perda de calor por evaporação cutânea nos animais mestiços $1 / 2$ Bôer $1 / 2$ Saanen foi maior que nos puros das raças Saanen e Alpina.

\section{Literatura Citada}

CENA, K.; MONTEITH, J.L. Transfer processes in animal coats. II. Convection and conduction. Procedure Royal Society, v. 188, p.395-411, 1975a.

CENA, K.; MONTEITH, J.L. Transfer processes in animal coats. III. Water vapor diffusion. Procedure Royal Society, v.188, p.413-423, 1975b.

SILVA, R.G. Bioclimatologia e melhoramento do gado leiteiro. Revista do Gado Holandês, n.148, p.5-12, 1988.

SILVA, R.G. Introdução à bioclimatologia animal. São Paulo: Nobel/FAPESP, 2000. 79p.

daSILVA, R.G.; LaSCALA JR., N.; LIMA FILHO, A.E. et al. Respiratory heat loss in the sheep: a comprehensive model. International Journal of Biometeorology, v.4, p.136-140, 2002 .

daSILVA, R.G.; STARLING, J.M.C. Evaporação cutânea e respiratória em ovinos sob altas temperaturas ambientes. Revista Brasileira de Zootecnia, v.32, n.6, p.1956-1961, 2003 (supl. 2)

daSILVA, R.G.; LaSCALA JR., N.; TONHATI, H. Radiative properties of the body surface of cattle and other animals. Transactions of the ASAE, v.46, p.913-918.

FINCH, V.A. Comparison of non-evaporative heat transfer in different cattle breeds. Australian Journal of Agricultural Research, v.36, p.497-508, 1985.

FINCH, V.A.; BENNETT, I.L.; HOLMES, C.R. Sweating response in cattle and its relation to rectal temperature, tolerance of sun and metabolic rate. Journal of Agricultural Science, v.99, p.479-487, 1982.

GEBREMEDHIN, K.G; CRAMER, C.O.; PORTER, W.P. Predictions and measurements of heat production and food and water requirements of Holstein calves in different environments. Transactions of the ASAE, v.3, p.715-720, 1981.

GEBREMEDHIN, K.G; PORTER, W.P.; CRAMER, C.O. Quantitative analysis of the heat exchange through the fur layer of Hostein calves. Transactions of the ASAE, p.188193,1983

GEBREMEDHIN, K.G.; BINXIN, WU. A model of evaporative cooling of wet skin surface and fur layer. Journal of Thermal Biology, v.26, p.537-545, 2001.

HARVEY, W.R. Least-aquares analysis of data with unequal subclass numbers. Beltaville: U.S.D.A., 1960. 157p (U.S.D.A, 20-8).

KERSLAKE, D.McK. The stress of hot environments. Cambridge: Cambridge University Press, 1972. 316p.
LEE, D.H.K. Manual of field studies on heat tolerance of domestic animals. ROMA: FAO, 1953. 161p.

LIMA, L.O.S.L. Aspectos genéticos da relação entre a taxa de sudação e a produção de leite em vacas da raça Holandesas. Jaboticabal: Universidade Estadual Paulista, 1991. 62p. Tese (Doutorado em Zootecnia) - Universidade Estadual Paulista, 1991.

LITTELL, R.C.; FREUND, R.J.; SPECTOR, P.C. SAS ${ }^{\circledR}$ system for linear models. 3.ed. Cary: 1991. 329pp.

MAIA, A.S.C.; SILVA, R.G.; BERTIPAGLIA, E.C.A. Características do pelame de vacas Holandesas em ambiente tropical: Um estudo genético e adaptativo. Revista Brasileira de Zootecnia, v.32, n.4, p. 843-853, 2003.

MAIA, A.S.C; DaSILVA, R.G.; BATTISTON, C.M. Respiratory heat loss of Holstein cows in a tropical environment. International Journal of Biometeorology, v.49, n.5, p.332-336, 2005.

MAIA, A.S.C; DaSILVA, R.G; BATTISTON, C.M. Sensible and latent heat loss from body surface of Holstein cows in a tropical environment. International Journal of Biometeorology (in press), 2005.

McLEAN, J.A. Measurement of cutaneous moisture vaporization from cattle by ventilated capsules. Journal of Physiology, v.167, p.417-426, $1963 \mathrm{a}$.

McLEAN, J.A. The partition of insensible losses of body weight in heat from cattle under various climatic conditions. Journal of Physiology, v.167, p.427-447, 1963b

OLIVEIRA, A.L. Aspectos genéticos de características adaptativas de cabras leiteiras em clima tropical. Jaboticabal: Universidade Estadual Paulista, 2004. 41p. Dissertação (Mestrado em Zootecnia) - Universidade Estadual Paulista, 2004.

STATISTICAL ANALYSES SYSTEM - SAS. SAS/STAT, User's guide: version 6.12, 4.ed. Cary: 1998. 842p.

SCHLEGER, A.V.; TURNER, H.G. Sweating rates of cattle in the field and their reaction to diurnal and seasonal changes. Australian Journal of Agricultural Reseacrh, v.16, p.92106,1965 .

STEVENS, D.G. A model of respiratory vapor loss in Holstein dairy cattle. Transactions of the ASAE, v.24 p.151-158, 1981.

TANEJA, G.C. Cutaneous evaporative losses in calves and its relationship with respiratory evaporative loss and skin and rectal temperatures. Journal of Agricultural Science, v.50, Part.1, p. $73-85,1958$.

TANEJA, G.C. Cutaneous evaporative loss measured from limited areas and its relationship with skin, rectal, and air temperatures. Journal of Agricultural Science, v.52, p.50-61,1959.

UDO, H.M.J. Hair coat characteristics in Friesian heifers in the Netherlands and Kenya. Wageningen: Mededelingen Landbouwhogeschool Wageningen, 1978. 135p. 